\title{
Real-world Evaluation of Tolerability, Safety and Efficacy of Rivastigmine Oral Solution in Patients with Mild to Moderate Alzheimer's Disease Dementia
}

\author{
Sun-Wung Hsieh ${ }^{1,2,3, *}$, Jui-Cheng Chen ${ }^{4,5,6 * *}$, Nai-Ching Chen $^{7,8}$, Kai-Ming Jhang ${ }^{9}$, Wenfu Wang ${ }^{9,10}$, \\ Yuan-Han Yang $2,3,11,12,13$ \\ ${ }^{1}$ Department of Neurology, Kaohsiung Municipal Siao-Gang Hospital, Kaohsiung Medical University, ${ }^{2}$ Department of Neurology, Kaohsiung Medical \\ University Hospital, Kaohsiung Medical University, ${ }^{3}$ Neuroscience Research Center, Kaohsiung Medical University, Kaohsiung, ${ }^{4}$ Department \\ of Neurology, China Medical University Hsinchu Hospital, ${ }^{5}$ Department of Neurology, China Medical University Hospital, ${ }^{6}$ School of Medicine, \\ China Medical University, Taichung, ${ }^{7}$ Department of Neurology, Kaohsiung Chang Gung Memorial Hospital, ${ }^{8}$ Department of Neurology, Chang \\ Gung University College of Medicine, Kaohsiung, ${ }^{9}$ Department of Neurology, Changhua Christian Hospital, ${ }^{10}$ Department of Holistic Wellness, \\ Ming Dao University, Changhua, ${ }^{11}$ Department of Neurology, Kaohsiung Municipal Ta-Tung Hospital, Kaohsiung Medical University Hospital, \\ ${ }^{12}$ Department of and Master's Program in Neurology, Faculty of Medicine, Kaohsiung Medical University, ${ }^{13}$ Chinese Mentality Protection \\ Association, Kaohsiung, Taiwan
}

\begin{abstract}
Objective: The purpose of this study is to investigate the safety, tolerability and efficacy of titrating dose of rivastigmine oral solution in patients with mild to moderate Alzheimer's disease (AD) in Taiwan.

Methods: We recruited 108 mild to moderate AD patients with Rivas ${ }^{\mathbb{R}}$ (rivastigmine oral solution $2 \mathrm{mg} / \mathrm{ml}$ ) treatment for 52 weeks. We recorded the demographic characteristics, initial cognition by mini-mental state examination (MMSE), initial global status by clinical dementia rating (CDR) with CDR-Sum of Boxes (CDR-SB), initial dose, and titrating dose at each visit. We investigated the adherence, proportion of possible side effects, optimal dose, and time to optimal dose. We demonstrated the proportion of cognitive decline and its possible risk factors.

Results: During the course, 9 patients discontinued the rivastigmine oral solution due to poor compliance or preference. Twelve out of 99 patients (12.1\%) reported possible side effects. Among 87 patients, the mean age was $77.2 \pm 9.0$ years ago with female predominant (65.2\%). The optimal dose was $3.6 \pm 1.4 \mathrm{ml}$ in average and $4 \mathrm{ml}(\mathrm{n}=31,35.6 \%)$ in mode. The duration to optimal dose was $12.5 \pm 10.2$ weeks and 24 weeks $(n=35,40.2 \%)$ in mode. It presented $25 \%$ with cognitive decline in MMSE, $27 \%$ with global function decline in CDR and $63 \%$ with global function decline in CDR-SB.

Conclusion: We demonstrated the clinical experience of rivastigmine oral solution in mild to moderate AD patients. It suggested rivastigmine oral solution $4 \mathrm{ml}$ is the optimal dose with 24 weeks to the optimal dose for at least one third of patients.
\end{abstract}

KEY WORDS: Rivastigmine; Alzheimer's disease; Dementia; Mini-mental state examination; Tolerability; Efficacy.

Received: June 30, 2020 / Revised: September 3, 2020

Accepted: October 16, 2020

Address for correspondence: Yuan-Han Yang

Department of Neurology, Kaohsiung Municipal Ta-Tung

Hospital, 68 Jhonghua 3rd Road, Cianjin District, Kaohsiung

80145, Taiwan

E-mail: yang1728@yahoo.com

ORCID: https://orcid.org/0000-0002-1699-4842

Wenfu Wang

Department of Neurology, Changhua Christian Hospital, 135,

Nanxiao St., Changhua 500, Taiwan

E-mail:w4239.tw@gmail.com

ORCID: https://orcid.org/0000-0002-3963-1649

*These authors contributed equally to this study as co-first authors.

\section{INTRODUCTION}

Alzheimer's disease (AD) is the commonest form of dementia affecting elderly people. The etiology of the disease is not clearly identified, but several mechanisms for the development of $\mathrm{AD}$ have been proposed. The cholinergic hypothesis is one of the proposed hypotheses. The deficiency of Acetylcholine (ACh) was found to lead to dysfunctional cholinergic signaling in the cortex and hip-

(ㄷ) This is an Open-Access article distributed under the terms of the Creative Commons Attribution Non-Commercial License (http://creativecommons.org/licenses/by-nc/4.0) which permits unrestricted non-commercial use, distribution, and reproduction in any medium, provided the original work is properly cited. 
pocampus, considering as the cause of cognitive impairment. Accordingly, Ach is the primary neurotransmitter facilitating learning and improving attention [1,2]. By far, different types of drugs used for cholinergic neurotransmission modification, and cholinesterase inhibitors remain the mainstay of treatment for mild to moderate AD by inhibiting the breakdown of released acetylcholine and enhancing the cholinergic neurotransmission [3-7].

Rivastigmine is a carbamate-type dual inhibitor of brain cholinesrterase, acetyl-cholinesterase (AChE) and butyl-cholinesterase (BuChE). It is characterized by penetrating the blood-brain barrier easily and targeting AChE and BuChE in the brain specifically, particularly in the hippocampus and cortex $[8,9]$. With the presence of rivastigmine, ACh hydrolysis is inhibited and levels of ACh are elevated in brain synapses. Rivastigmine had shown its efficacy in the symptomatic treatment of improving or maintaining cognitive function, daily living activities, behaviors, and global dementia symptoms in patients with mild to moderate AD and Parkinson's disease dementia [10-12]. The therapeutic dosage of rivastigmine was suggested to titrate from the lower to higher dose for the better clinical response [13]. Due to the cholinergic deficit, tolerability, and treatment response potential, it may be worthwhile keeping high-dose cholinesterase inhibition in reserve [14]. However, considering the anticipation of the gastrointestinal adverse events (nausea, vomiting, diarrhea) associated with cholinesterase inhibitors, it might be dose dependent causing some extent of withdraw medication. Furthermore, the incidence of adverse effects depends on duration of enzyme inhibition and the extent of daily fluctuations in enzyme activity [15]. It is concluded that reducing daily fluctuations in the rivastigmine pharmacokinetic profile contributes decrease in fluctuations in the extent of enzyme inhibition and improvement in overall tolerability. For this reason, Transdermal patches offer many advantages over conventional oral medications.

Oral rivastigmine is available as capsules $(1.5,3.0,4.5$, and $6.0 \mathrm{mg}$ ) and a bioequivalent oral solution $(2 \mathrm{mg} / \mathrm{ml})$, administered twice daily [16]. Various trials have showed that gastrointestinal adverse events can be tolerated by the slow titration $[17,18]$. The real-world clinical experience of rivastigmine oral solution is less discussed. Our study predicts that adjusting lower dose to higher dose rivastigmine with oral solution can improve the patient's and caregiver's compliance as well as decrease the medical discontinue rate. Herein we conducted the observational study investigating the safety, tolerability and efficacy of titrating dose of rivastigmine oral solution in patients with mild to moderate AD in Taiwan.

\section{METHODS}

\section{Study Population}

We conducted an open label, non-comparative and observational study to investigate the safety, tolerability and efficacy of Rivast $^{\circledR}$ (rivastigmine oral solution $2 \mathrm{mg} / \mathrm{ml}$ ) in mild to moderate AD dementia patients in Taiwan. We recruited patients who met the criteria for $A D$ in neurological out-patient departments in three medical centers in Taiwan (Kaohsiung Chang Gung Memorial Hospital, China Medical University Hospital, and Changhua Christian Hospital). The diagnose of AD was based on the Diagnostic and Statistical Manual of Mental Disorder 4th edition (DSM-IV) criteria and National Institute of Neurological Disorders and Stroke (NINCDS)-Alzheimer's Disease and Related Disorders Association (ADRDA) criteria [19,20]. All the patients underwent a brain imaging survey and a set of blood screening tests, such as complete blood count, renal function, liver function, vitamin B12, folic acid, cortisol level and serologic test of syphilis, to exclude out the possibility of vascular or other type of dementia [21]. Psychometrics were administered for evaluating the global function by Clinical Dementia Rating (CDR) [22] with CDR-Sum of Boxes (CDR-SB) [22], and cognitive function by Mini-Mental State Examination (MMSE) [23]. We recorded the demographic data of the patients, including age, sex, body heights, body weights, body mass index (BMI), education duration, and glomerular filtration rate (GFR). For this study, all practices were carried out in accordance with the Helsinki Declaration, and were approved by Changhua Christian Hospital Institutional Review Board (CCH IRB No. 190501). All participants, or their legal representatives, provided written informed consent before entering the study.

\section{Inclusion and Exclusion Criteria}

The inclusion criteria for this study were as follows, (1) Patients having a clinical diagnosis of mild to moderate $A D$, fulfilling the DSM-IV criteria for dementia and NINCDSADRDA diagnostic criteria for probable AD. (2) Cognitive impairment demonstrated by neuropsychiatric tests, with 
an MMSE $<27$ and CDR $\geq 0.5$. (3) Generalized normal neurological examinations except impaired cognitive function. (4) No structural brain abnormalities to the diagnosis of AD. (5) The memory and cognitive impairment are not attributable to any medical conditions or medications. (6) Exclusion of other neurodegenerative diseases. (7) The patients or their attorney are able to understand the objectives of the study and signed the informed consent. The exclusion criteria were as follows, (1) Patients with known hypersensitivity to any other cholinesterase inhibitors. (2) Patients with clinical evidence of any medical/ neurological/psychiatric disorders which in opinion of investigators are likely to interfere with the study. (3) Patients who have a known or suspected history (within past year) of alcoholism, drug misuse or dementia secondary to alcohol abuse. (4) Cardiac disease potentially resulting in syncope, near syncope or other alterations of mental status. In addition, the following conditions should lead to exclusion: bradycardia less than 50 beats per minute, atrioventricular block. (5) Patients who have received investigational new drug within past three months.

\section{Recommended Rules for Titrating Rivast ${ }^{\circledR}$ (Rivastigmine Oral Solution $2 \mathrm{mg} / \mathrm{ml}$ )}

Titrating dose of Rivast ${ }^{\circledR}$ (rivastigmine oral solution 2 $\mathrm{mg} / \mathrm{ml}$ ) for AD patients was practiced by neurologists in each visit at 4th, 8th, 12th, 24th week after initial rivastigmine oral solution dose in the Neurological out-patient departments (OPD) following the recommended rules as Table 1. In real clinical practice, the initial dose of rivastigmine oral solution was started by neurologists according to their clinical experiences and the patients' condition. The neurologists adjusted the dose according to the tolerability reported by patients or their family members at each OPD follow-up. The patients reported the prescribed dose of rivastigmine oral solution in each OPD visit at 4th, 8th, 12th, 24th week. The prescribed dose of rivastigmine oral solution was recorded as optimal dose for the maximal tolerable prescribing dose maintaining for at least 3 months. We recorded the time to optimal dose from the onset time.

\section{Evaluation of Safety, Tolerability and Efficacy of Titrating Dose of Rivast ${ }^{\circledR}$ (Rivastigmine Oral Solution 2 mg/ml)}

We investigated and reported the adherence, proportion of possible side effects, optimal dose, and time to optimal dose throughout the initial 24 weeks of treating with rivastigmine oral solution at 24 weeks. The observational study will continue to 52 weeks eventually. We investigated the proportion of cognitive decline by MMSE, global function decline by CDR with $\mathrm{CDR}-\mathrm{SB}$, and risk factors to cognitive/global function decline. The study procedure and flowchart is demonstrated in Figure 1. The clinicians reported the prescribed dose of rivastigmine oral solution in initial OPD and each OPD visit at 4th, 8th, 12 th, and 24th week for the patients. The prescribed dose of rivastigmine oral solution was recorded as optimal dose for the maximal tolerable prescribing dose maintaining for at least 3 months. We recorded the time to optimal dose for patients. Cognitive decline by MMSE was defined as the amount of decreasing MMSE scores $\geq 3$ in one year. Global function decline by CDR was defined as progression in CDR level in one year. Global function decline by CDR-SB was defined as increasing in CDR-SB scores in one year. We calculated the correlation of optimal dose and time to optimal dose with demographic variables. We demonstrated the proportion of cognitive/global function decline and the possible risk factors.

\section{Statistical Analysis}

Statistical analyses were performed with the Statistical Package for the Social Sciences (SPSS version 19.0; IBM Co., Armonk, NY, USA). We presented the demographic data, including age, sex, body heights, body weights, BMI,

Table 1. Recommended rules for titrating Rivast ${ }^{\circledR}$ (rivastigmine oral solution)

\begin{tabular}{|c|c|c|c|c|c|c|c|c|c|c|c|c|}
\hline \multirow{2}{*}{ Dose } & \multicolumn{12}{|c|}{ Week } \\
\hline & \multicolumn{3}{|c|}{ Base-line } & \multicolumn{3}{|c|}{ 4th } & \multicolumn{3}{|c|}{ 8th } & \multirow{2}{*}{$\frac{12 \text { th }}{3}$} & \multirow{2}{*}{$\frac{24 \text { th }}{3}$} & \multirow{2}{*}{$\frac{52 \mathrm{nd}}{3}$} \\
\hline $\mathrm{AM}(\mathrm{ml})$ & 0.5 & 1 & 1 & 1.5 & 1.5 & 2 & 2 & 2.5 & 2.5 & & & \\
\hline $\mathrm{PM}(\mathrm{ml})$ & 1 & 1 & 1.5 & 1.5 & 2 & 2 & 2.5 & 2.5 & 3 & 3 & 3 & 3 \\
\hline Daily dose (mg) & 3 & 4 & 5 & 6 & 7 & 8 & 9 & 10 & 11 & 12 & 12 & 12 \\
\hline
\end{tabular}

$1 \mathrm{ml}=2 \mathrm{mg}$; AM, ante meridiem; PM, post meridiem. 


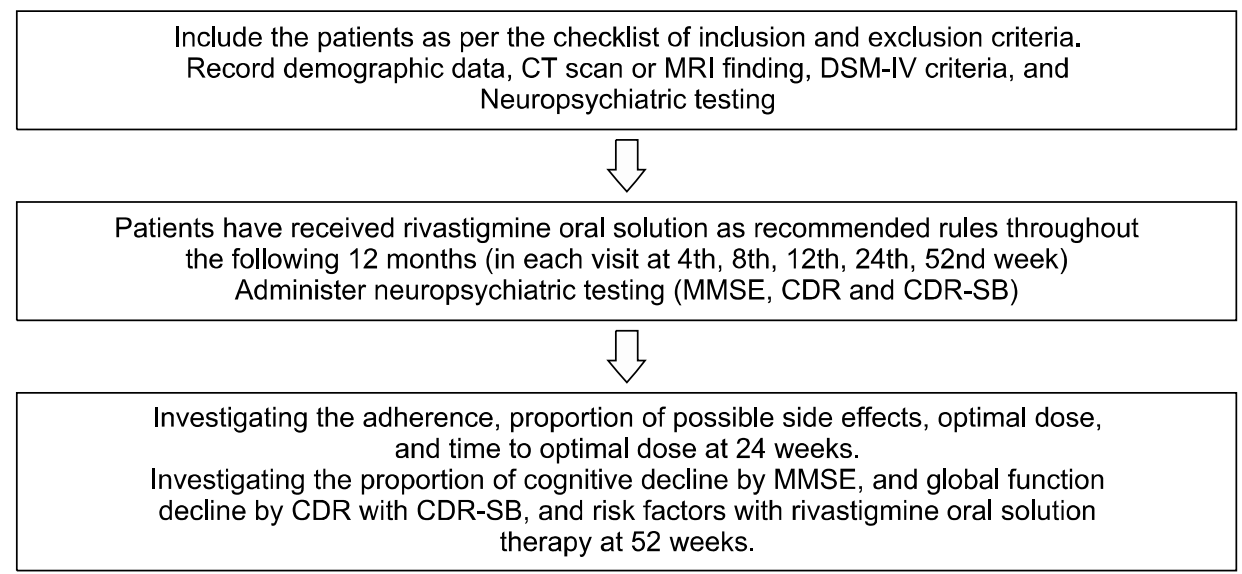

Fig. 1. Study procedure and flowchart.

CT, computed tomography; MRI, magnetic resonance imaging; DSM-IV, Diagnostic and Statistical Manual of Mental Disorder 4th edition; MMSE, mini-mental state examination; CDR, clinical dementia rating; CDR-SB, Clinical Dementia Rating-Sum of Boxes.

education duration and GFR at study onset. Cognitive function by MMSE and global function by CDR with CDR-SB were presented at study onset and after full therapy for 52 weeks. To assess the safety and tolerability of rivastigmine oral solution, we calculated the proportion of patients who did not complete the study for the possible side effects or discontinuation of therapy. We recorded the initial dose, titrating dose at each OPD visit at 4th, 8th, 12th, and 24th week after study onset, optimal dose and duration to optimal dose. The data were presented as maximal value, minimal value, mean (standard deviation), mode (numbers, percentages), and median for continuous variables and numbers (percentages) for categorical variables. We calculated the correlation of optimal dose, time to optimal dose and initial dose with demographic variables using Pearson correlation test. To assess the efficacy of rivastigmine oral solution, cognitive function by MMSE and global function by CDR with CDR-SB were compared before and after rivastigmine oral solution therapy. We determined the significant factors associated with cognitive decline by MMSE and global function decline by CDR with CDR-SB using Student's $t$ test. All statistical tests were two-tailed, and a $p$ value of 0.05 was considered to show significance with $95 \%$ confidence interval.

\section{RESULTS}

\section{Description of Studies for Tolerability and Safety}

We recruited 108 patients with mild to moderate AD in the study. During the course, there were 9 patients dis-
Table 2. Demographic characteristics $(n=87)$

\begin{tabular}{lccc}
\hline \multicolumn{1}{c}{ Variable } & Min & Max & Value \\
\hline Age $(\mathrm{yr})$ & 55 & 93 & $77.2 \pm 9.0$ \\
Sex, female & & & $57(65.2)$ \\
Education $(\mathrm{yr})$ & 0 & 20 & $6.5 \pm 5.2$ \\
Height $(\mathrm{cm})$ & 134.6 & 185 & $154.8 \pm 9.7$ \\
Body weight $(\mathrm{kg})$ & 41.6 & 90 & $57.8 \pm 10.7$ \\
BMl & 16.5 & 38.8 & $23.8 \pm 4.7$ \\
GFR & 6.5 & 152 & $72.1 \pm 29.9$ \\
Initial MMSE & 10 & 27 & $15.0 \pm 6.9$ \\
Initial CDR-SB & 0.5 & 18 & $5.7 \pm 3.9$ \\
\hline
\end{tabular}

Values are presented as mean \pm standard deviation or number $(\%)$. $\mathrm{BMI}$, body mass index; GFR, glomerular filtration rate; MMSE, minimental state examination; CDR-SB, Clinical Dementia Rating-Sum of Boxes.

continued the rivastigmine oral solution due to poor compliance or preference, including 5 for poor compliance and 4 for preference of oral capsule or patch. The rest 99 patients were evaluated for the tolerability, safety and efficacy of rivastigmine oral solution. There were 12 patients reporting the possible side effects and discontinuing the therapy, including 2 for dizziness, 4 for nausea/vomiting, 1 for somnolence, 1 for hallucination, 1 for diarrhea, 1 for hypotension, and 2 for unexpected mortality due to malignancy or bleeding. The proportion of possible side effects was $12.1 \%$ (12 out of 99 patients). There were 87 patients having the rivastigmine oral solution therapy for full 52 months, including 4 patients with sinus bradycardia but continuously taking medication. We evaluated the tolerability, safety and efficacy of rivastigmine oral solution therapy. The overall adherence was $80.6 \%$ (87 out 
Table 3. Dose of each visit, optimal dose and time to optimal dose $(n=87)$

\begin{tabular}{llccrc}
\hline \multicolumn{1}{c}{ Variable } & Min & Max & $\begin{array}{c}\text { Mean } \pm \text { standard } \\
\text { deviation }\end{array}$ & Mode (number, \%) & Median \\
\hline Initial dose $(\mathrm{ml})$ & 0.25 & 6 & $2.0 \pm 1.4$ & $1(33,37.9)$ & 2 \\
V1 dose (ml) (4th wek) & 0.5 & 6 & $2.6 \pm 1.4$ & $2(37,42.5)$ & 2 \\
V2 dose (ml) (8th week) & 0.5 & 6 & $2.7 \pm 1.4$ & $2(33,37.9)$ & 2 \\
V3 dose (ml) (12th week) & 0.5 & 6 & $3.0 \pm 1.4$ & $2(30,34.5)$ & 3 \\
V4 dose (ml) (24th week) & 0.5 & 6 & $3.6 \pm 1.4$ & $4(31,35.6)$ & 4 \\
Optimal dose (ml) & 0.5 & 6 & $3.6 \pm 1.4$ & $4(31,35.6)$ & 4 \\
Time to optimal dose (wk) & 0 & 24 & $12.5 \pm 10.2$ & $24(35,40.2)$ & 12 \\
\hline
\end{tabular}

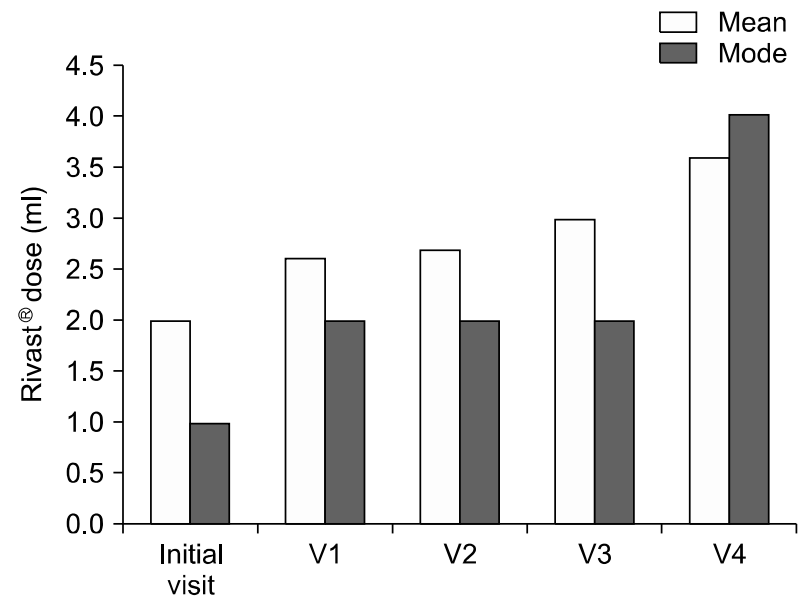

Fig. 2. The mean and mode dose during each visit.

of 108 patients) in our study.

The demographic characteristic of our study was demonstrated in Table 2. The mean age in our study was $77.2 \pm$ 9.0 years ago (range $55-93$ years old) with female predominant ( 57 females, $65.2 \%$ ). The mean education duration was $6.5 \pm 5.2$ years (range $0-20$ years). The mean body weights was $57.8 \pm 10.7 \mathrm{~kg}$ (range $41.6-90 \mathrm{~kg}$ ). The mean BMI was $23.8 \pm 4.7$ (range 16.5-38.8). The mean GFR was $72.1 \pm 29.9$ (range 6.5-152). The mean initial MMSE was $15.0 \pm 6.9$ (range 10-27). The mean CDR-SB was $5.7 \pm 3.9$ (range $0.5-18$ ).

\section{The Optimal Dose and Time to Optimal Dose}

The adjusted dose of each visit, optimal dose and time to optimal dose were demonstrated in Table 3 and Figure 2. The initial mean dose was $2.0 \pm 1.4 \mathrm{ml}$ (range $0.25-6$ $\mathrm{ml})$ and the initial mode dose was $1 \mathrm{ml}(\mathrm{n}=33,37.9 \%)$. The mean dose in 24th week was $3.6 \pm 1.4 \mathrm{ml}$ (range $0.5-$ $6 \mathrm{ml})$ and the mode dose in 24th weeks was $4 \mathrm{ml}(\mathrm{n}=31$, $35.6 \%$ ). The mean optimal dose was $3.6 \pm 1.4 \mathrm{ml}$ (range $0.5-6 \mathrm{ml})$ and the mode of optimal dose was $4 \mathrm{ml}(\mathrm{n}=$

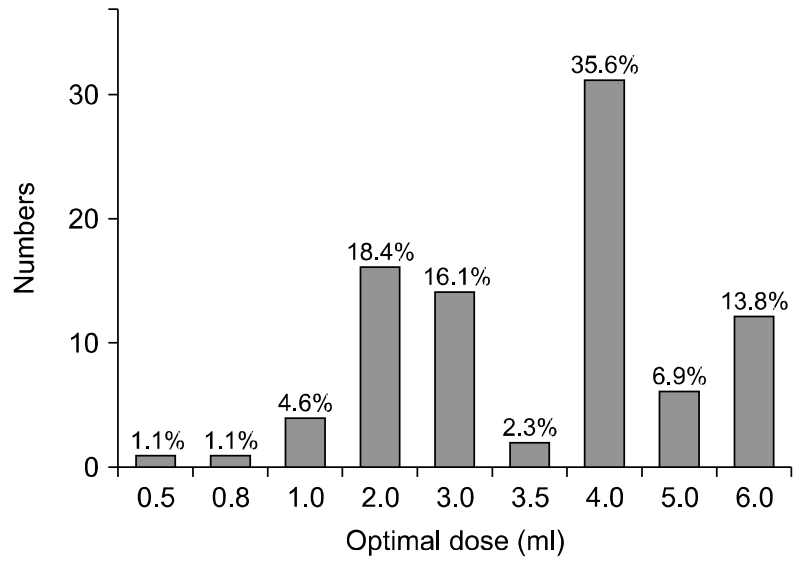

Fig. 3. The distribution of numbers associated with optimal dose.

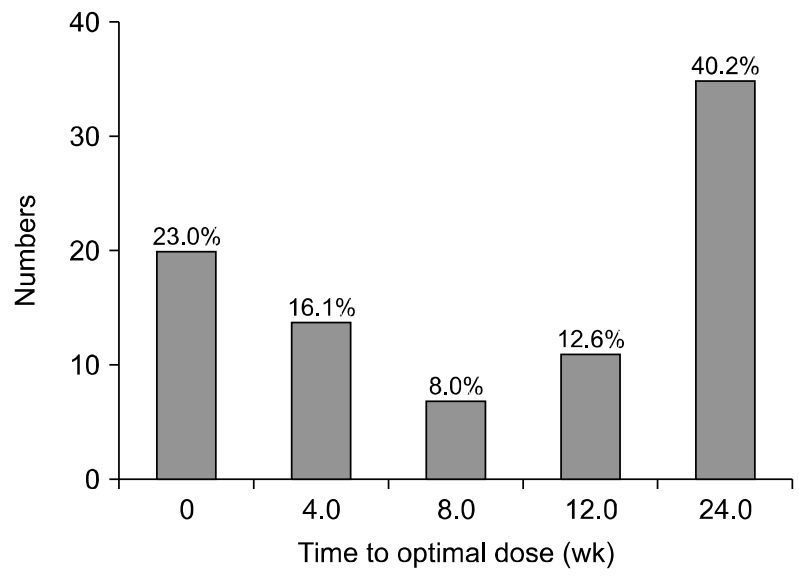

Fig. 4. The distribution of numbers associated with time to optimal dose.

$31,35.6 \%)$. The mean duration to optimal dose was $12.5 \pm$ 10.2 weeks (range $0-24$ weeks) and the mode of duration to optimal dose was 24 weeks ( $n=35,40.2 \%$ ). The distributions of numbers in associated with optimal dose and time to optimal dose were demonstrated in Figures 3 and 4 . The correlation of demographic variables with dose-related variables was shown in Table 4. The age was 
Table 4. Correlation of optimal dose, time to optimal dose and initial dose with demographic variables

\begin{tabular}{cccccccrrrr}
\hline Variable & & Age & Height & BW & BMI & GFR & $\begin{array}{r}\text { Initial } \\
\text { MMSE }\end{array}$ & $\begin{array}{r}\text { Final } \\
\text { MMSE }\end{array}$ & $\begin{array}{c}\text { Initial } \\
\text { CDR-SB }\end{array}$ & $\begin{array}{c}\text { Final } \\
\text { CDR-SB }\end{array}$ \\
\hline Optimal dose (ml) & $\mathrm{r}$ & $-0.213^{*}$ & 0.012 & 0.044 & 0.019 & 0.025 & 0.042 & 0.095 & 0.006 & 0.052 \\
& $p$ & 0.048 & 0.915 & 0.690 & 0.867 & 0.818 & 0.702 & 0.430 & 0.954 & 0.662 \\
Time to optimal & $\mathrm{r}$ & -0.138 & 0.018 & 0.017 & 0.013 & 0.048 & 0.064 & 0.106 & -0.059 & -0.100 \\
dose (wk) & $p$ & 0.204 & 0.873 & 0.876 & 0.909 & 0.656 & 0.561 & 0.379 & 0.587 & 0.395 \\
Initial dose (ml) & $\mathrm{r}$ & -0.040 & -0.081 & -0.050 & -0.008 & -0.038 & -0.094 & -0.064 & 0.160 & 0.203 \\
& $p$ & 0.714 & 0.460 & 0.655 & 0.945 & 0.726 & 0.390 & 0.594 & 0.140 & 0.082 \\
\hline
\end{tabular}

BW, body weights; BMI, body mass index; GFR, glomerular filtration rate; MMSE, mini-mental state examination; CDR-SB, Clinical Dementia Rating-Sum of Boxes; r, correlation coefficient.

${ }^{*} p<0.05$, statistic significant.

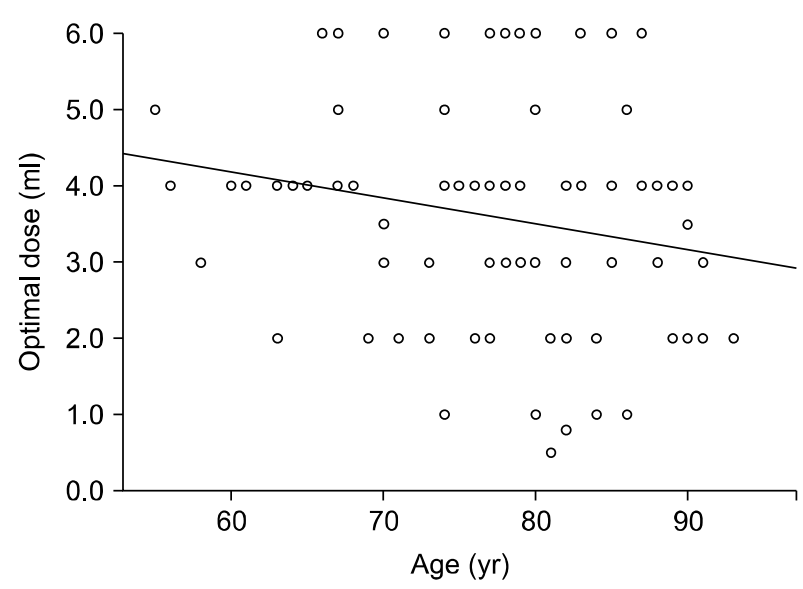

Fig. 5. Correlation of age with optimal dose. r: $-0.213, p=0.048$

negatively correlated with optimal dose $(r=-0.213, p=$ 0.048) (Fig. 5). Difference of optimal dose, time to optimal dose, and initial dose in sex was shown in Table 5 and it revealed no significant difference.

\section{Efficacy of Rivast ${ }^{\circledR}$ (Rivastigmine Oral Solution)}

The cognitive function by MMSE and global function by CDR with CDR-SB before and after rivastigmine oral solution therapy was demonstrated in Table 6. The mean initial MMSE was $15.0 \pm 6.9$. The mean final MMSE was $14.5 \pm 7.8$. There was no significant difference in MMSE before and after rivastigmine oral solution therapy ( $p=$ 0.656). There were 24 patients $(27.6 \%)$ with CDR0.5, 45 patients (51.7\%) with CDR1 and 18 patients (20.6\%) with CDR2. After having rivastigmine oral solution for 52 months, there were 18 patients (20.7\%) with CDR0.5, 33 patients (37.9\%) with CDR1 and 17 patients (19.5\%) with CDR2 and 6 patients $(6.9 \%)$ with CDR3. There was no significant difference in CDR before and after rivastigmine
Table 5. Difference of optimal dose, time to optimal dose, and initial dose in sex

\begin{tabular}{lccc}
\hline \multicolumn{1}{c}{ Variable } & Male $(\mathrm{n}=30)$ & Female $(\mathrm{n}=57)$ & $p$ value \\
\hline Optimal dose $(\mathrm{ml})$ & $3.7 \pm 1.4$ & $3.5 \pm 1.5$ & 0.573 \\
Time to optimal dose $(\mathrm{wk})$ & $12.9 \pm 9.8$ & $12.2 \pm 10.4$ & 0.755 \\
Initial dose $(\mathrm{ml})$ & $1.9 \pm 1.1$ & $2.1 \pm 1.5$ & 0.427 \\
\hline
\end{tabular}

Values are presented as mean \pm standard deviation. $p<0.05$, statistic significant.

Table 6. The cognitive function in MMSE, CDR, and CDR-SB in initial visit and after rivastigmine oral solution therapy

\begin{tabular}{lccc}
\hline Variable & Initial & Final & $p$ value \\
\hline MMSE & $15.0 \pm 6.9$ & $14.5 \pm 7.8$ & 0.656 \\
CDR & $\mathrm{n}=87$ & $\mathrm{n}=74$ & \\
CDR0.5 & $24(27.6)$ & $18(20.7)$ & 0.154 \\
CDR1 & $45(51.7)$ & $33(37.9)$ & \\
CDR2 & $18(20.6)$ & $17(19.5)$ & \\
CDR3 & $0(0)$ & $6(6.9)$ & \\
CDR-SB & $5.7 \pm 3.9$ & $7.1 \pm 5.0$ & $0.042^{*}$ \\
\hline
\end{tabular}

Values are presented as mean \pm standard deviation or number (\%). MMSE, mini-mental state examination; $C D R$, clinical dementia rating; CDR-SB, Clinical Dementia Rating-Sum of Boxes.

${ }^{*} p<0.05$, statistic significant.

oral solution therapy $(p=0.154)$. The mean initial CDR-SB was $5.7 \pm 3.9$. The mean final CDR-SB was $7.1 \pm$ 5.0. There was significant difference in CDR-SB before and after rivastigmine oral solution therapy $(p=0.042)$.

In our study, there were $25 \%$ ( 18 out of 71 with complete MMSE evaluation) patients having cognitive decline in MMSE, $27 \%$ (20 out of 73 with complete CDR evaluation) patients having global function decline in CDR and $63 \%$ (46 out of 73 with complete CDR-SB evaluation) patients having global function decline in CDR-SB (Table 7-9). The initial dose, optimal dose, and time to optimal dose were not significantly associated with cognitive/global 
Table 7. Possible factors associated with cognitive/global function decline by MMSE

\begin{tabular}{lccc}
\hline \multicolumn{1}{c}{ Variable } & $\begin{array}{c}\text { Cognitive decline } \\
\text { by MMSE }(\mathrm{n}=18)\end{array}$ & $\begin{array}{c}\text { Cognitive preserved } \\
\text { by MMSE }(\mathrm{n}=53)\end{array}$ & $p$ value \\
\hline Age $(\mathrm{yr})$ & $76.3 \pm 9.6$ & $76.6 \pm 9.5$ & 0.924 \\
Sex, female & $7(38.9)$ & $37(69.8)$ & $0.020^{*}$ \\
Education $(\mathrm{yr})$ & $8.9 \pm 6.1$ & $6.4 \pm 4.9$ & 0.077 \\
GFR & $81.7 \pm 31.1$ & $71.9 \pm 28.6$ & 0.224 \\
Initial dose $(\mathrm{ml})$ & $2.0 \pm 0.9$ & $1.7 \pm 1.4$ & 0.411 \\
Optimal dose $(\mathrm{ml})$ & $3.1 \pm 1.3$ & $2.7 \pm 1.3$ & 0.221 \\
Time to optimal dose $(\mathrm{wk})$ & $5.8 \pm 5.4$ & $4.9 \pm 4.6$ & 0.508 \\
Initial MMSE & $14.5 \pm 6.3$ & $16.1 \pm 6.8$ & 0.385 \\
Initial CDR-SB & $7.4 \pm 3.8$ & $4.8 \pm 3.7$ & $0.016^{*}$ \\
\hline
\end{tabular}

Values are presented as mean \pm standard deviation or number (\%).

MMSE, mini-mental state examination; CDR, clinical dementia rating; CDR-SB, Clinical Dementia Rating-Sum of Boxes.

${ }^{*} p<0.05$, statistic significant.

Table 8. Possible factors associated with cognitive/global function decline by CDR

\begin{tabular}{lccc}
\hline \multicolumn{1}{c}{ Variable } & $\begin{array}{c}\text { Global function decline } \\
\text { by CDR }(\mathrm{n}=20)\end{array}$ & $\begin{array}{c}\text { Global function preserved } \\
\text { by CDR }(\mathrm{n}=53)\end{array}$ & $p$ value \\
\hline Age $(\mathrm{yr})$ & $79.1 \pm 10.2$ & $75.5 \pm 9.0$ & 0.181 \\
Sex, female & $14(70.0)$ & $31(58.5)$ & 0.367 \\
Education $(\mathrm{yr})$ & $5.6 \pm 5.3$ & $7.4 \pm 5.3$ & 0.196 \\
GFR & $80.3 \pm 28.5$ & $71.4 \pm 29.4$ & 0.248 \\
Initial dose $(\mathrm{ml})$ & $1.9 \pm 1.6$ & $1.8 \pm 1.3$ & 0.777 \\
Optimal dose $(\mathrm{ml})$ & $3.2 \pm 1.5$ & $2.7 \pm 1.3$ & 0.215 \\
Time to optimal dose $(\mathrm{wk})$ & $4.2 \pm 4.6$ & $5.4 \pm 4.8$ & 0.328 \\
Initial MMSE & $12.1 \pm 6.5$ & $16.9 \pm 6.3$ & $0.009^{*}$ \\
Initial CDR-SB & $6.0 \pm 3.4$ & $5.4 \pm 4.1$ & 0.519 \\
\hline
\end{tabular}

Values are presented as mean \pm standard deviation or number (\%).

MMSE, mini-mental state examination; CDR, clinical dementia rating; CDR-SB, Clinical Dementia Rating-Sum of Boxes.

${ }^{*} p<0.05$, statistic significant.

Table 9. Possible factors associated with cognitive/global function decline by CDR-SB

\begin{tabular}{lccc}
\hline \multicolumn{1}{c}{ Variable } & $\begin{array}{c}\text { Global function decline } \\
\text { by CDR-SB }(\mathrm{n}=46)\end{array}$ & $\begin{array}{c}\text { Global function preserved } \\
\text { by CDR-SB }(\mathrm{n}=27)\end{array}$ & $p$ value \\
\hline Age $(\mathrm{yr})$ & $78.7 \pm 8.8$ & $72.8 \pm 9.2$ & $0.008^{*}$ \\
Sex, female & $28(60.9)$ & $18(66.7)$ & 0.769 \\
Education $(\mathrm{yr})$ & $7.1 \pm 5.6$ & $6.7 \pm 4.8$ & 0.737 \\
GFR & $75.0 \pm 26.6$ & $70.8 \pm 33.3$ & 0.548 \\
Initial dose $(\mathrm{ml})$ & $1.9 \pm 1.3$ & $1.8 \pm 1.4$ & 0.820 \\
Optimal dose $(\mathrm{ml})$ & $3.5 \pm 1.4$ & $3.5 \pm 1.4$ & 0.883 \\
Time to optimal dose $(\mathrm{wk})$ & $12.3 \pm 9.9$ & $14.3 \pm 11.0$ & 0.415 \\
Initial MMSE & $15.4 \pm 6.4$ & $16.0 \pm 7.1$ & 0.719 \\
Initial CDR-SB & $5.5 \pm 3.4$ & $5.7 \pm 4.6$ & 0.822 \\
\hline
\end{tabular}

Values are presented as mean \pm standard deviation or number (\%).

MMSE, mini-mental state examination; CDR, clinical dementia rating; CDR-SB, Clinical Dementia Rating-Sum of Boxes.

${ }^{*} p<0.05$, statistic significant.

function decline by MMSE, CDR and CDR-SB. Female was associated with less cognitive decline by MMSE (38.9\% vs. $69.8 \%, p=0.02)$ (Fig. 5). Higher initial CDR-SB was associated with cognitive decline by MMSE
(7.4 \pm 3.8 vs. $4.8 \pm 3.7, p=0.016$ ) (Table 7). Lower initial MMSE scores was associated with global function decline by CDR $(12.1 \pm 6.5$ vs. $16.9 \pm 6.3, p=0.009)$ (Table 8 ). Older age was associated with global function decline by 
CDR-SB $(78.7 \pm 8.8$ vs. $72.8 \pm 9.2, p=0.008)$. Initial MMSE and CDR-SB showed no significant difference in determining the global function decline by CDR-SB (Table 9).

\section{DISCUSSION}

We demonstrated the real-world clinical experience of rivastigmine oral solution $(2 \mathrm{mg} / \mathrm{ml})$ in mild to moderate AD patients in Taiwan. It suggested rivastigmine oral solution $4 \mathrm{ml}$ is the optimal dose with 24 weeks to reach to the optimal dose for at least one third of patients. During the course, 9 patients discontinued the rivastigmine oral solution due to poor compliance or preference. Twelve out of 99 patients $(12.1 \%)$ reported possible side effects. The overall adherence was $80.6 \%$ (87/108) in our study.

This is the first observational study reporting the safety and tolerability of rivastigmine oral solution in mild to moderate $\mathrm{AD}$ dementia patients in Asian population. Similar study was conducted in Phoenix, Arizona, USA, comparing the safety and tolerability of novel rivastigmine transdermal patch (a 24-h single application of a $9.5 \mathrm{mg} / 24-\mathrm{h} ; 10 \mathrm{~cm}^{2} ; 18 \mathrm{mg}$ dose load) with rivastigmine oral solution (single $3 \mathrm{mg}$ dose) in 30 healthy elderly subjects (MMSE > 27, 13 males, mean 67.7 years old, and mean $73.6 \mathrm{~kg}$ in body weights) [16]. Adverse events reported after either patch or oral solution administration were most frequently associated with the gastrointestinal system and nervous system, consistent with the cholinomimetic actions of rivastigmine. The occurrence of gastrointestinal-related adverse events (nausea, vomiting) was lower with the patch (6 subjects, $20 \%$ ) than with the oral solution (10 subjects, $33 \%$ ). The occurrence of nervous system-related adverse events (headache and dizziness) was $8(27 \%)$ with the patch and $10(33 \%)$ with the oral treatment. Our study focused on the practicing titrating dose in clinical, showing less reporting adverse events than the previous study. Meanwhile, we concluded that lower optimal dose is considered in AD patients with older age to avoid the possible side events.

There was no difference in cognition function by MMSE and global function decline by CDR before and after rivastigmine oral solution therapy in our study, but it presented $25 \%$ with cognitive decline by MMSE, $27 \%$ with global function decline by CDR. More than two thirds of patients had cognitive preservation in MMSE or CDR with rivastigmine oral solution therapy for 1 year. While in CDR-SB, it showed significant change in CDR-SB before and after rivastigmine oral solution therapy and up to $63 \%$ of patients with global function decline in CDR-SB. This is because $A D$ is a progressive neurodegenerative disease. MMSE is used extensively in clinical and research settings to measure cognitive impairment as well as cognitive outcomes to cholinesterase inhibitors therapy $[24,25]$. CDR-SB is a qualitative instrument for assessing the global function and staging the severity of dementia. Studies have adopted this global severity score as therapeutic outcome $[26,27]$. In spite of the limited case numbers we enrolled in the study, CDR-SB could give a new insight into global function evaluation in AD patients under therapy. In considering the possible risk factors in determining the cognitive/global function decline, we concluded male sex and higher initial CDR-SB are risk factors for cognitive decline by MMSE, lower initial MMSE is the risk factor for global function decline by CDR and older age is the risk factor for global function decline by CDR-SB. Compared to the previous study discussing the efficacy of oral rivastigmine in Taiwan, Chen et al. [28] reported $41.3 \%$ of AD patients had improvement in cognition by MMSE and $63.5 \%$ in global status by CDR-SB. The clinically MMSE improving group had a significantly higher rivastigmine concentration, lower initial MMSE, lower initial CDR-SB scores and presence of APOE $\varepsilon 4$-carriers. Higher education was significantly associated with clinical improvement in global status by CDR-SB. Although therapeutic response rate varies from 20 to $60 \%$, concentrations of rivastigmine may benefit cognitive function of $\mathrm{AD}$ patients. In spite of lack rivastigmine concentration in our study, it demonstrated higher proportion of cognitive preservation with rivastigmine oral solution therapy for $\mathrm{AD}$ patients, suggesting the oral solution form with the titrating method may benefit in reaching the dose-dependent effect.

Although our study provided the useful information in optimal dose and time to optimal dose of rivastigmine oral solution therapy in real-world practice, there were limitations in our study. Firstly, we did not characterize the plasma pharmacokinetics, bioavailability and metabolite NAP226-90 (inactive pharmacologically) of rivastigmine following oral solution administrations. These profiles might help to understand the fluctuations of the drug concentration in association with the overall tolerability, safety and efficacy. Secondly, Apolipoprotein E gene (ApoE) 
was not genotyped in AD patients in our study. Apolipoprotein E4 (ApoE4) is the most prevalent genetic risk factor of $\mathrm{AD}$ [29] with its numerous implications in processes of crosstalk with beta-amyloid $(A \beta)$ and effect on lipid metabolism and inflammation [30-32]. ApoE4 is a promising $\mathrm{AD}$ therapeutic target for its role in mediating the processes [33]. Thirdly, we did not enrolled the predictive factors that might have impact on the tolerability, safety and efficacy of rivastigmine in AD patients, such as baseline cardiovascular risk factors, psychological factors, medication, lifestyle, environment [34], diet habit, nutritious status, socio-economic status and family support. Fourthly, we did not enroll the information of behavioral and psychological symptoms of dementia (BPSD). Nearly $90 \%$ of AD patients presented with BPSD, leading to independence reduction and incapability of completing daily activities. These might have impacts on medication adherence, tolerability and efficacy [35]. The overall adherence was $80.6 \%$ in our study. The frequencies of non-adherence of medication in dementia patients varied considerably across studies in real world [36]. In a cohort study, Stoehr and colleagues [37] concluded the non-adherence rate was $10.7 \%$ among cognitive impairment elders aged more than 65 years. The greatest rate of nonadherence was 38\% in one prospective cohort study using electronic monitoring [38]. From one case control study, adherence frequencies using 'pill counts' ranged from 17-100\% among AD patients [39]. The adherence frequencies of oral solution were rarely discussed.

Various neuro-inflammatory processes and cytokines had been proved to have the impact on the pathology of AD [40-42]. Currently, acetylcholinesterase inhibitors are believed to have anti-inflammatory properties $[43,44]$. Evidences indicated $\mathrm{M} 2 \mathrm{~b}$ macrophages may have crucial role in improving nerve injuries and brain diseases. The M2b macrophages polarization was gradually used as an inflammatory biomarker in its role of diseases of nervous system and AD $[45,46]$. Future studies focusing on the macrophage polarization pattern in $\mathrm{AD}$ patients with rivastigmine oral solution therapy help to clarify the anti-inflammatory properties of acetylcholinesterase inhibitors and promote the evaluation of therapeutic efficacy more precisely.

The response of rivastigmine to cognitive domains in AD patients, such as memory, language, attention or executive function, vary with study design and the effects re- main inconclusive [47-49]. It needs further investigations about the efficacy of rivastigmine oral solution in various cognitive domains. In consideration of treating AD, BPSD is an important issue. BPSD lead to poor outcomes, distress among patients and caregivers, earlier placement in nursing homes, long-term hospitalization, misuse of medication, and increased health care costs $[35,50]$. Whether the oral solution benefits more than the conventional oral capsule in management of BPSD requires further evaluation. Current treatment strategy in AD involves multiple approaches combining pharmacological and non-pharmacological intervention. Making maintenance and establishment of a strong therapeutic alliance to physician, patient, and caregiver is crucial [51]. Accordingly, rivastigmine oral solution in combination with non-pharmacological intervention is encouraging to enhance its efficacy in cognitive improvement and functional abilities.

We demonstrated the clinical experience of rivastigmine oral solution in $\mathrm{AD}$ patients. It suggested rivastigmine oral solution $4 \mathrm{ml}$ is the optimal dose with 24 weeks to the optimal dose for at least one third of patients. This study predicts that adjusting treatment dose with rivastigmine oral solution can improve the patient's and caregiver's compliance and decrease medical discontinue rate.

\section{- Acknowledgments}

This study was supported by the Department of Neurology, Kaohsiung Municipal Ta-Tung Hospital, Kaohsiung, Taiwan (kmtth-107-004), Neuroscience Research Center, Kaohsiung Medical University Research Center Grant (KMUTC108B01), Kmhk-104-035, Kmhk-108-033, S-108-009, DMR-109-073, CMUHCH-DMR-109-009, MOST1082314-B-039-003 and 108-CCH-PRJ-174.

\section{a Conflicts of Interest}

No potential conflict of interest relevant to this article was reported.

\section{- Author Contributions}

Conceptualization: Wenfu Wang. Data acquisition: Jui-Cheng Chen, Nai-Ching Chen, Kai-Ming Jhang. Formal analysis: Yuan-Han Yang. Supervision: Wenfu Wang, Yuan-Han Yang. Writing - original draft, review \& editing: Sun-Wung Hsieh. 


\section{ORCID}

Sun-Wung Hsieh https://orcid.org/0000-0003-3952-7718 Jui-Cheng Chen https://orcid.org/0000-0003-3987-4816 Nai-Ching Chen https://orcid.org/0000-0003-2684-0377 Kai-Ming Jhang https://orcid.org/0000-0001-9525-9944 Wenfu Wang https://orcid.org/0000-0002-3963-1649 Yuan-Han Yang https://orcid.org/0000-0002-1699-4842

\section{REFERENCES}

1. Gauthier S. Advances in the pharmacotherapy of Alzheimer's disease. CMAJ 2002;166:616-623.

2. Wang HY, Lee DH, D'Andrea MR, Peterson PA, Shank RP, Reitz AB. beta-Amyloid(1-42) binds to alpha7 nicotinic acetylcholine receptor with high affinity. Implications for Alzheimer's disease pathology. I Biol Chem 2000;275:56265632.

3. Practice guideline for the treatment of patients with Alzheimer's disease and other dementias of late life. American Psychiatric Association. Am J Psychiatry 1997; 154(5 Suppl):1-39.

4. Small GW, Rabins PV, Barry PP, Buckholtz NS, DeKosky ST, Ferris $\mathrm{SH}$, et al. Diagnosis and treatment of Alzheimer disease and related disorders. Consensus statement of the American Association for Geriatric Psychiatry, the Alzheimer's Association, and the American Geriatrics Society. JAMA 1997;278: 1363-1371.

5. Doody RS, Stevens JC, Beck C, Dubinsky RM, Kaye JA, Gwyther L, et al. Practice parameter: management of dementia (an evidence-based review). Report of the Quality Standards Subcommittee of the American Academy of Neurology. Neurology 2001; 56:1154-1166.

6. Cummings JL, Frank JC, Cherry D, Kohatsu ND, Kemp B, Hewett L, et al. Guidelines for managing Alzheimer's disease: part 1. Assessment. Am Fam Physician 2002;65:2263-2272.

7. Cummings JL, Frank JC, Cherry D, Kohatsu ND, Kemp B, Hewett L, et al. Guidelines for managing Alzheimer's disease: part II. Treatment. Am Fam Physician 2002;65:2525-2534.

8. Grossberg GT, Stahelin HB, Messina JC, Anand R, Veach J. Lack of adverse pharmacodynamic drug interactions with rivastigmine and twenty-two classes of medications. Int J Geriatr Psychiatry 2000;15:242-247.

9. Polinsky RJ. Clinical pharmacology of rivastigmine: a newgeneration acety/cholinesterase inhibitor for the treatment of Alzheimer's disease. Clin Ther 1998;20:634-647.

10. Bullock R. The clinical benefits of rivastigmine may reflect its dual inhibitory mode of action: an hypothesis. Int J Clin Pract 2002; 56:206-214.

11. Onor ML, Trevisiol M, Aguglia E. Rivastigmine in the treatment of Alzheimer's disease: an update. Clin Interv Aging 2007;2:17-32.

12. Rösler M, Anand R, Cicin-Sain A, Gauthier S, Agid Y,
Dal-Bianco $\mathrm{P}$, et al. Efficacy and safety of rivastigmine in patients with Alzheimer's disease: international randomised controlled trial. BMJ 1999;318:633-638.

13. Farlow $\mathrm{M}$, Anand $\mathrm{R}$, Messina J Jr, Hartman R, Veach J. $A$ 52-week study of the efficacy of rivastigmine in patients with mild to moderately severe Alzheimer's disease. Eur Neurol 2000:44:236-241.

14. Small G, Bullock R. Defining optimal treatment with cholinesterase inhibitors in Alzheimer's disease. Alzheimers Dement 2011;7:177-184.

15. Imbimbo BP. Pharmacodynamic-tolerability relationships of cholinesterase inhibitors for Alzheimer's disease. CNS Drugs 2001; 15:375-390.

16. Lefèvre G, Pommier F, Sedek G, Allison M, Huang HL, Kiese $\mathrm{B}$, et al. Pharmacokinetics and bioavailability of the novel rivastigmine transdermal patch versus rivastigmine oral solution in healthy elderly subjects. J Clin Pharmacol 2008;48: 246-252.

17. Cumbo E, Ligori LD. Differential effects of current specific treatments on behavioral and psychological symptoms in patients with Alzheimer's disease: a 12-month, randomized, open-label trial. J Alzheimers Dis 2014;39:477-485.

18. Blanco-Silvente L, Castells X, Saez M, Barceló MA, GarreOlmo J, Vilalta-Franch J, et al. Discontinuation, efficacy, and safety of cholinesterase inhibitors for Alzheimer's disease: a meta-analysis and meta-regression of 43 randomized clinical trials enrolling 16106 patients. Int I Neuropsychopharmacol 2017;20:519-528.

19. American Psychiatric Association. Diagnostic and statistical manual of mental disorders, fourth edition (DSM-IV). Washington: American Psychiatric Association; 1994. p. 143-147.

20. McKhann G, Drachman D, Folstein M, Katzman R, Price D, Stadlan EM. Clinical diagnosis of Alzheimer's disease: report of the NINCDS-ADRDA Work Group under the auspices of Department of Health and Human Services Task Force on Alzheimer's Disease. Neurology 1984;34:939-944.

21. Yang YH, Fuh JL, Mok VC. Vascular contribution to cognition in stroke and Alzheimer's disease. Brain SCi Adv 2018;4: 39-48.

22. Morris JC. The Clinical Dementia Rating (CDR): current version and scoring rules. Neurology 1993;43:2412-2414.

23. Folstein MF, Folstein SE, McHugh PR. "Mini-mental state". A practical method for grading the cognitive state of patients for the clinician. J Psychiatr Res 1975;12:189-198.

24. Wattmo C, Wallin AK, Londos E, Minthon L. Predictors of long-term cognitive outcome in Alzheimer's disease. Alzheimers Res Ther 2011;3:23.

25. Birks J, Grimley Evans J, lakovidou V, Tsolaki M, Holt FE. Rivastigmine for Alzheimer's disease. Cochrane Database Syst Rev 2009; (2):CD001191.

26. O’Bryant SE, Lacritz LH, Hall J, Waring SC, Chan W, Khodr $\mathrm{ZG}$, et al. Validation of the new interpretive guidelines for the clinical dementia rating scale sum of boxes score in the na- 
tional Alzheimer's coordinating center database. Arch Neurol 2010;67:746-749.

27. O’Bryant SE, Waring SC, Cullum CM, Hall J, Lacritz L, Massman PJ, et al. Staging dementia using Clinical Dementia Rating Scale Sum of Boxes scores: a Texas Alzheimer's research consortium study. Arch Neurol 2008;65:1091-1095.

28. Chen TH, Chou MC, Lai CL, Wu SJ, Hsu CL, Yang YH. Factors affecting therapeutic response to rivastigmine in Alzheimer's disease patients in Taiwan. Kaohsiung J Med Sci 2017;33: 277-283.

29. Strittmatter WJ, Roses AD. Apolipoprotein E and Alzheimer's disease. Annu Rev Neurosci 1996; 19:53-77.

30. Michaelson DM. APOE \&4: the most prevalent yet understudied risk factor for Alzheimer's disease. Alzheimers Dement 2014;10:861-868.

31. Holtzman DM, Herz J, Bu G. Apolipoprotein E and apolipoprotein E receptors: normal biology and roles in Alzheimer disease. Cold Spring Harb Perspect Med 2012;2:a006312.

32. Di Battista AM, Heinsinger NM, Rebeck GW. Alzheimer's disease genetic risk factor APOE-E4 also affects normal brain function. Curr Alzheimer Res 2016;13:1200-1207.

33. Safieh M, Korczyn AD, Michaelson DM. ApoE4: an emerging therapeutic target for Alzheimer's disease. BMC Med 2019; 17:64.

34. Cerejeira J, Lagarto L, Mukaetova-Ladinska EB. Behavioral and psychological symptoms of dementia. Front Neurol 2012;3:73.

35. Hosseini-Sharifabad A, Rabbani M, Seyed-Yousefi Y, Safavi M. Magnesium increases the protective effect of citicoline on aluminum chloride-induced cognitive impairment. Clin Psychopharmacol Neurosci 2020;18:241-248.

36. Smith D, Lovell J, Weller C, Kennedy B, Winbolt M, Young C, et al. A systematic review of medication non-adherence in persons with dementia or cognitive impairment. PLoS One 2017;12:e0170651.

37. Stoehr GP, Lu SY, Lavery L, Bilt JV, Saxton JA, Chang CC, et al. Factors associated with adherence to medication regimens in older primary care patients: the Steel Valley Seniors Survey. Am J Geriatr Pharmacother 2008;6:255-263.

38. Insel K, Morrow D, Brewer B, Figueredo A. Executive function, working memory, and medication adherence among older adults. J Gerontol B Psychol Sci Soc Sci 2006; 61:P102 P107.

39. Cotrell V, Wild K, Bader T. Medication management and adherence among cognitively impaired older adults. J Gerontol Soc Work 2006; 47:31-46.
40. Heneka MT, Carson MJ, El Khoury J, Landreth GE, Brosseron F, Feinstein DL, et al. Neuroinflammation in Alzheimer's disease. Lancet Neurol 2015;14:388-405.

41. Van Eldik LJ, Carrillo MC, Cole PE, Feuerbach D, Greenberg $\mathrm{BD}$, Hendrix JA, et al. The roles of inflammation and immune mechanisms in Alzheimer's disease. Alzheimers Dement ( $N$ Y) 2016;2:99-109.

42. Kinney JW, Bemiller SM, Murtishaw AS, Leisgang AM, Salazar AM, Lamb BT. Inflammation as a central mechanism in Alzheimer's disease. Alzheimers Dement (N Y) 2018:4:575590.

43. Goschorska M, Baranowska-Bosiacka I, Gutowska I, Tarnowski M, Piotrowska K, Metryka E, et al. Effect of acety/cholinesterase inhibitors donepezil and rivastigmine on the activity and expression of cyclooxygenases in a model of the inflammatory action of fluoride on macrophages obtained from THP-1 monocytes. Toxicology 2018;406-407:9-20.

44. Shifrin H, Nadler-Milbauer M, Shoham S, Weinstock M. Rivastigmine alleviates experimentally induced colitis in mice and rats by acting at central and peripheral sites to modulate immune responses. PLoS One 2013;8:e57668.

45. Wang LX, Zhang SX, Wu HJ, Rong XL, Guo J. M2b macrophage polarization and its roles in diseases. J Leukoc Biol 2019;106:345-358.

46. Hsieh SW, Huang LC, Chang YP, Hung CH, Yang YH. M2b macrophage subset decrement as an indicator of cognitive function in Alzheimer's disease. Psychiatry Clin Neurosci 2020;74:383-391.

47. Frankfort SV, Appels BA, de Boer A, Tulner LR, van Campen $\mathrm{JP}, \mathrm{Koks} \mathrm{CH}$, et al. Identification of responders and reactive domains to rivastigmine in Alzheimer's disease. Pharmacoepidemiol Drug Saf 2007; 16:545-551.

48. McKeith I, Del Ser T, Spano P, Emre M, Wesnes K, Anand R, et al. Efficacy of rivastigmine in dementia with Lewy bodies: a randomised, double-blind, placebo-controlled international study. Lancet 2000;356:2031-2036.

49. Gauthier S, Juby A, Rehel B, Schecter R. EXACT: rivastigmine improves the high prevalence of attention deficits and mood and behaviour symptoms in Alzheimer's disease. Int J Clin Pract 2007;61:886-895.

50. Preuss UW, Wong JW, Koller G. Treatment of behavioral and psychological symptoms of dementia: a systematic review. Psychiatr Pol 2016;50:679-715.

51. Yang YH, Liscic R, Dominguez J. Framework of treating Alzheimer's dementia. Brain Sci Adv 2019;5:82-93. 